\title{
Lumen
}

Selected Proceedings from the Canadian Society for Eighteenth-Century Studies

\section{« Maître en l'art de plaire » ou « bel esprit » ? Le comte Andrei Chouvalov vu par la police et le beau monde de Paris en 1777-1781}

\section{Dzianis Kandakou}

Volume 35, 2016

URI : https://id.erudit.org/iderudit/1035920ar

DOI : https://doi.org/10.7202/1035920ar

Aller au sommaire du numéro

Éditeur(s)

Canadian Society for Eighteenth-Century Studies / Société canadienne d'étude du dix-huitième siècle

ISSN

1209-3696 (imprimé)

1927-8284 (numérique)

Découvrir la revue

Citer cet article

Kandakou, D. (2016). « Maître en l'art de plaire » ou « bel esprit » ? Le comte Andrei Chouvalov vu par la police et le beau monde de Paris en 1777-1781. Lumen, 35, 47-62. https://doi.org/10.7202/1035920ar 


\title{
«Maître en l'art de plaire » ou «bel esprit»? Le comte Andrei Chouvalov vu par la police et le beau monde de Paris en 1777-1781
}

\author{
DZIANIS KANDAKOU \\ Université d'État de Polotsk
}

Le comte Andrei Petrovitch Chouvalov est sans doute le plus fameux des écrivains francophones russes des Lumières. Au sein de la République des Lettres, l'auteur de l'Epître à Ninon de l'Enclos dialogue avec Voltaire, La Harpe, Dorat ou encore Parny et Saint-Lambert ${ }^{1}$. Cependant ses contacts avec le beau monde parisien, cet arbitre rigoureux du bon goût, sont peu explorés. Pour rétablir le réseau de relations du comte Chouvalov, pour comparer sa réputation littéraire avec celle qu'il se fait dans les grands salons de Paris, il serait utile de se référer aux rapports de la police qui se veut omnisciente. Les enquêtes du contrôle des étrangers permettent de voir et d'entendre ce qui se passe et ce qui se dit au sein de l'assemblée diplomatique, pendant les dîners privés ou

1. Sur ce sujet voir Д. Кобеко, «Ученик Вольтера граф А.П. Шувалов», Русский архив [Dmitri Kobeko, «Élève de Voltaire. Le comte A.P. Chouvalov», Archives russes], 1881, t. 3, p. 241-290; Jean-François de La Harpe, Letters to the Shuvalovs, éd. de C. Todd, Oxford, Studies on Voltaire and the Eighteenth Century, 1973; П. Заборов, «Русско-французские поэты XVIII века», Многоязычие и литературное творчество [Petr Zaborov, "Poètes franco-russes du XVIII ${ }^{\mathrm{e}}$ siècle ", Multilinguisme et création littéraire], Leningrad, Nauka, 1981, p. 61-105; Petr Zaborov, «Le rôle des écrivains franco-russes dans la formation du "mirage russe" au XVIII siècle", dans Serguei Karp et Larry Wolff (éd.), Le mirage russe au XVIII siècle, FerneyVoltaire, Centre international d'études du XVIII siècle, 2001, p. 193-201; Alexandre Stroev, «Les filleuls de Voltaire», dans Jean Bessière et Judit Maar (éd.), Histoire de la littérature et jeux d'échange entre centres et la périphéries: Les identités relatives des littératures, Paris, L’Harmattan, 2010, p. 181-19o; Elena Gretchanaïa, "Je vous parlerai la langue de l'Europe... » : La francophonie en Russie (XVIII'-XIX ${ }^{e}$ siècles), Berne, Peter Lang, 2012, p. 52-64. 
les spectacles. C'est le travail des inspecteurs Buhot, Lechenetier Delongpré ou Bossonet ${ }^{2}$ qui notent une fois par semaine toutes les apparitions mondaines des diplomates, donnent des portraits succincts des étrangers de haut rang. À leurs rapports très souvent secs et factuels s'ajoutent ceux du département des mœurs, riches en détails piquants, présentant un large panorama du demi-monde 3 . Très probablement, dans les années 1770-1780, les inspecteurs de la partie des étrangers et de la partie des mœurs collaborent pour surveiller les grands personnages venus d'ailleurs dont Andrei Petrovitch Chouvalov.

Nous allons aussi présenter une pièce de vers «oubliée » signée par le comte Chouvalov. Malgré son caractère stéréotypé, cette poésie fugitive peut en dire long sur les relations mondaines de son auteur. Les documents en question datent du troisième séjour parisien du seigneur russe qui dure de novembre 1777 à juillet 1781 . Les deux autres visites (1756-1758 et 1764-1766) préparent sa future gloire mais ne laissent pas beaucoup de traces.

À la fin des années 1770, le comte Andrei Chouvalov est déjà plus qu'un des élèves de Voltaire. Chambellan, sénateur, directeur de la Banque d'État des assignats, il est aussi une figure marquante des lettres européennes francophones. Ses poésies vantent en premier lieu les progrès de la Russie en sciences, en métiers et en arts. Parmi ces derniers, «l'art de plaire » est l'un des plus importants et, selon Dorat, le comte Chouvalov en est maître ${ }^{4}$. Comment fait-il pour mériter ce titre flatteur?

2. Sur leurs fonctions et leurs activités, voir Jean-François Dubost, «Les étrangers à Paris au siècle des Lumières ", dans Daniel Roche (éd.), La ville promise. Mobilité et accueil à Paris (fin XVII'-début XIX siècle), Paris, Fayard, 2000, p. 221-288; Rachel Couture, "Inspirer la crainte, le respect et l'amour du public": les inspecteurs de police parisiens, 1740-1789", thèse de doctorat, sous la dir. de P. Bastien et V. Milliot, Montréal \Caen, UQAM \Université de Caen Basse Normandie, mars 2013.

3. Au sujet de ce département, voir Érica-Marie Benabou, La prostitution et la police des moeurs au XVIII siècle, Paris, Perrin, 1987; Nina Kushner, Erotic Exchanges: The World of Elite Prostitution in Eighteenth-Century Paris, Ithaca, Cornell University Press, 2013.

4. «Toi, qui loin du Nord engourdi, / Viens chez nous, maître en l'art de plaire... ", c'est l'incipit du Billet à M. le comte de Sch..l.. [Chouvalov] par ClaudeJoseph Dorat publié dans le Mercure de France du 16 octobre 1779, p. 99. 


\section{Le comte russe dans le beau monde et le demi-monde}

Lorsque le comte Andrei Chouvalov arrive à Paris pour la première fois, il est encore adolescent, et ses manières, ses connaissances, son français lui valent d'emblée l'attention particulière et la sympathie générale. Il est présenté au roi et au dauphin, il est reçu chez l'abbé de Bernis, la marquise de l'Hôpital et des ministres étrangers. D'après le témoignage du chargé d'affaires russe Fedor Dmitrievitch Bekhteev, toute la bonne société parisienne est d'accord que le jeune comte Chouvalov «est un homme fait» et "qu'il est charmant" ».

En 1764, il revient à Paris avec sa femme, ce qui ne l'empêche pas de prendre connaissance du monde galant où «la demoiselle Clairon, ex-actrice du Théâtre Français, guida ses premiers pas $^{6}$ ». On doit cette déclaration ultérieure à un inspecteur de police et nous ne pouvons ni la prouver ni la contester. Les fameux rapports sur les femmes galantes destinés à Louis XV taisent la liaison éventuelle entre le comte Andrei Chouvalov et la belle Clairon. Cependant ils mentionnent à quelques reprises un certain «M. Choaloffe» qui courtise les demoiselles Robbe, Grécourt et Dangeville en 1765, et la marquise de Bellegarde en 1766 . Tout porte à croire qu'il s'agit d'Ivan Ivanovitch Chouvalov, l'oncle de notre héros, un personnage très bien connu à Paris à cette époque. En ce qui concerne le neveu, il complète le programme que se propose tout étranger de distinction: après l'aventure amoureuse avec l'actrice, il fait le pèlerinage classique à Ferney en septembre 1765 et marque sa visite par une épître au patriarche. Cette pièce en vers voit le jour très vite: avec une réponse badine et bienveillante de Voltaire et une réplique du comte, elle est publiée au Journal encyclopédique du $1^{\text {er }}$ octobre 1765 . Ainsi, Andrei Petrovitch Chouvalov devient-il finalement un homme fait, un homme fait pour Paris.

En novembre 1777, son retour à la capitale française mérite un grand rapport. Sans oublier de donner quelques éléments biographiques et généalogiques, d'ailleurs bien incorrects, l'inspecteur de police met en relief la richesse et surtout la générosité du seigneur russe:

5. Lettre de F. D. Bekhteev au comte M. I. Vorontsov, Paris, le 22 août 1757 (Archives Vorontsov, t. 6, Moscou, W. Gautier, 1873, p. 206).

6. AMAE, Contrôle des étrangers, vol. 18, f. $82 \mathrm{r}^{\circ}$.

7. Voir Paris sous Louis XV: rapports des inspecteurs de police au roi..., éd. de C. Piton, Paris, Mercure de France, 1905-1914, t. 2, p. 164-165; t. 3, p. 54, 138 et 163. 
M. le comte de Schuwalow est parti de St. Pétersbourg au mois de juin pour venir en droiture à Paris, mais des indispositions qu'ont éprouvées ces époux, et la chute de la voiture des enfants dans une rivière en Courlande, qui faillit leur faire perdre la vie, sont causes de la longueur du voyage.

Cet accident arriva dans un endroit écarté où il n'était pas possible de trouver une retraite pour soigner les enfants [...] M. le comte dépêcha un homme à cheval pour demander l'hospitalité à un gentilhomme courlandais, qui refusa de les recevoir. Ils furent obligés de faire encore deux lieues et furent reçus à bras ouverts par un curé qui les traita pendant trois jours de la manière la plus noble.

M. le comte, pour reconnaître la façon d'agir de ce curé, lui fit présent d'une bague de diamants de 2000 roubles qu'il ne voulait point accepter, mais le premier la laissa sur la table ${ }^{8}$.

Mais ce n'est pas cet épisode qui attire l'attention de la police au comte Chouvalov. Ce sont ses connaissances à la capitale, surtout dans le monde diplomatique, qui le placent, ainsi que son épouse, la comtesse Ekaterina Petrovna, parmi les personnages les plus fréquents de la chronique du contrôle des étrangers entre novembre 1777 et juillet 1781. Dans cet intervalle de temps, les Chouvalov sont évoqués 150 (!) fois, ce qui est impressionnant vu que les travaux d'inspecteurs de police sont essentiellement consacrés aux ministres étrangers.

Les mouches déclarent que les époux ne fréquentent pas les mêmes sociétés ${ }^{9}$ et l'analyse de leurs rapports semble le prouver. Le tableau 1 (voir l'annexe) montre que les portes de la maison du maréchal de Biron, toujours «très brillante par le nombre d'étrangers », sont grandes ouvertes aux Chouvalov, tant au comte qu'à la comtesse. La deuxième maison la plus fréquentée est celle du baron de Schoenfeld, ministre de Saxe en France, où l'on trouve surtout Madame la comtesse (trois fois) et une fois le couple. Enfin, les Chouvalov sont admis à la société de la marquise Du Deffand, qui d'ailleurs trouve la comtesse «indo-

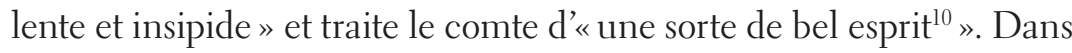
les autres salons tenus par les dames de distinction, la police ne remarque que Ekaterina Petrovna: chez la duchesse d'Enville, la

8. AMAE, Contrôle des étrangers, vol. $18, \mathrm{f} .82 \mathrm{v}^{\circ}-83 \mathrm{r}^{\circ}$.

9. Voir le travail du 23 juillet 1779 (ibid., vol. 30 , f. 4 Or $^{\circ}$ ).

10. Lettre de la marquise Du Deffand à Horace Walpole, le 8 mars 1778, Horace Walpole's Correspondence, éd. de W.S. Lewis et W.H. Smith, New-Haven, Yale University Press, 1961, t. VII, p. 26. 
duchesse de Luxembourg, la princesse de Guéménée ou encore chez la duchesse de Mirepoix et le prince polonais August Kazimierz Sulkowski.

D'ailleurs, ces données ne sont ni exhaustives ni complètement correctes. Les mémorialistes de l'époque sont unanimes: les Russes vivent dans le Paris des Lumières en parfaite intelligence, comme une seule famille. Donc les Chouvalov durent se rendre chez le ministre de Russie, le prince Ivan Bariatinski, plus de trois fois durant trois ans et neuf mois. L'inventaire des visites effectuées dans les foyers de la noblesse française mérite quelques ajouts et corrections. Le 26 février 1778, la comtesse d'Hunolstein écrit à Ivan Ivanovitch Chouvalov que son neveu «fait toutes les semaines une banque chez la maréchale de Luxembourg, cette complaisance lui fait un grand mérite auprès de toutes dames ». La marquise de Barbentane avoue au même correspondant qu'elle voit Ekaterina Petrovna "plus que [elle] le peu[t]», la comtesse russe soupe et joue plusieurs fois chez la fille de la marquise, la comtesse de Hunolstein en printemps $177^{11}$. D'après le journal de la baronne Natalia Mikhailovna Stroganova, la comtesse Chouvalova fréquente les maisons de la duchesse de Chartres et de la duchesse de La Vallière ${ }^{12}$. Aussi peut-on affirmer que les Chouvalov sont reçus dans toutes les grandes maisons de Paris conformément à leur condition et jouissent de tous les agréments de la société.

Parmi ces derniers, le comte Chouvalov n'oublie pas les plus doux. Fort de l'expérience précédente avec la demoiselle Clairon, il renoue ses liens avec le monde galant. Le rapport du 23 juillet 1779 signale :

M. le comte de Schouwalow, Russe, [...] après avoir connu le monde galant, s'est enfin attaché à la demoiselle Asselin, danseuse de l'Opéra. Il lui a loué un appartement dans la rue de Chabanois qu'il a fait meubler magnifiquement, et il est très assidu auprès de cette Laïs.

Ces amants ont cependant été en brouille ces jours derniers; et la demoiselle Asselin ayant renvoyé le comte, lui avait jeté ses diamants par

11. Voir les lettres de la comtesse d'Hunolstein et de la marquise de Barbentane à Ivan Ivanovitch Chouvalov datées de 1777 et 1778: OP РНБ [Département des manuscrits, Bibliothèque nationale de Russie], Fr. Q.IV.207, f. 123-126, 126-130, 135-136, 143-144.

12. Voir Е.П. Гречаная, Когда Россия говорила по-франиузски: русская литература на франиузском языке (XVIII - первая половина ХІХ века) [E.P. Gretchanaïa, Quand la Russie parlait français: la littérature russe d'expression française (XVIII ${ }^{-}$première moitié du XIX siècle)], Moscou, IMLI RAN, 2010, p. 147. 
la fenêtre, avait cassé la porcelaine qu'il lui avait donnée, lui avait défendu sa porte, et paraissait décidée à ne plus le recevoir; mais ce dernier à force de soumissions a fléchi le cœur de sa maîtresse, et pour lui témoigner sa reconnaissance, il lui a fait 6ooo louis de rente, et les diamants sont maintenant chez le Sr. Lempereur pour être rétablis.

Dans le premier séjour que ce seigneur a fait ici, il a vécu avec la demoiselle Clairon, ex-actrice du Théâtre Français, et a fait pour elle une très grande dépense. Comme il est extrême, la demoiselle Asselin pourra le mener loin ${ }^{13}$.

L'auteur du rapport s'avère un mauvais prophète. Le crédit (dans tous les sens du mot) du comte Chouvalov est trop solide, même pour les appétits de la demoiselle Asselin. Il évite la ruine et reçoit la complaisance de la danseuse. Les liaisons deviennent si fortes avec le temps que, à en croire les informateurs de la police, la demoiselle Asselin et son époux le chevalier d'Alose partent pour la Russie avec les Chouvalov ${ }^{14}$.

Cette histoire ressemble aux autres histoires galantes notées par les inspecteurs Meusnier et Marais, responsables du département des mœurs entre 1748 et 1780 . Comme tout étranger de distinction avant et après lui, le comte Chouvalov entretient une actrice, lui fait des cadeaux somptueux. Plus qu'un plaisir ou une extravagance, c'est une nécessité mondaine. Comme Nina Kushner le montre, le demi-monde du XVIII ${ }^{\text {e }}$ siècle fonctionne comme une société parallèle au grand monde parisien et à la cour ${ }^{15}$. Une liaison avec une femme entretenue permet de partager quelques «délices» avec les personnages de très haut rang, de les côtoyer, voire de rivaliser avec eux. Quinze ans avant le séjour du comte Chouvalov, son compatriote le prince Andrei Mikhailovitch Belosselski réussit à enlever la demoiselle Lacour, danseuse à l'Opéra, au duc de La Vallière (un nom que nous allons évoquer plus d'une fois...) pour un collier de 15000 livres, une robe de velours garnie de queue de martre, cent pistoles par mois et 300 louis de pot-de-vin ${ }^{16}$.

Mais revenons au comte Chouvalov et la demoiselle Asselin. C'est une liaison qui devait rendre le Russe assez célèbre et... un peu mépri-

13. AMAE, Contrôle des étrangers, vol. 30, f. $39 \mathrm{r}^{\circ}-4 \mathrm{Or}^{\circ}$.

14. Voir le rapport du 6 juillet 178 ( (ibid., vol. 39 , f. $5 \mathrm{r}^{\circ}-\mathrm{v}^{\circ}$ ).

15. Nina Kushner, Erotic Exchanges, op. cit., p. 4-6 et suivantes.

16. Paris sous Louis XV, op. cit., t. 1, p. 301-303. 
sable aux yeux de la société parisienne. La demoiselle Asselin n’est pas des plus respectables. Outre sa conduite scandaleuse dont le rapport de police ci-dessus fait preuve, elle est trop connue pour ses rôles bien frivoles: dans le ballet pantomime Les Petits-Riens monté à l'Opéra le 11 juin 1778 , elle joue une bergère déguisée en berger qui finit par «se dévoiler ${ }^{{ }^{17}}$. Deux ans plus tôt, elle se trouve mêlée dans un procès retentissant concernant l'héritage de sa sœur aînée ${ }^{18}$. Le départ avec une telle personne à Saint-Pétersbourg, s'il était connu, rendrait le comte Chouvalov ridicule dans les salons de Paris.

\section{La politique dans le salon du comte Chouvalov}

Si les inspecteurs de police négligent parfois les sorties des Chouvalov, les visites qu'ils reçoivent sont surveillées de très près. Il y a certainement une mouche (voire plusieurs) qui opère dans la maison et fait des rapports minutieux sur toutes les entrées dignes d'attention. Celles-ci sont vraiment nombreuses: entre août 1778 et juillet 1781 , la police compte 84 réceptions à la maison des Chouvalov. Le comte et la comtesse partagent ces visites par moitié: 42 pour Andrei Petrovitch et 42 pour Ekaterina Petrovna ${ }^{19}$. Les Russes sont certainement les bienvenus et les principaux habitués. Les mouches remarquent que « $\mathrm{M}$. le comte de Schouwalow [...] donne un dîner tous les mercredis pour ses compatriotes » et que «le ministre plénipotentiaire de Russie dîne chez M. le comte Schouwalow toutes les fois qu'il n'est pas engagé ailleurs ${ }^{20} »$.

À l'opposé, les Français semblent ignorer la maison des Chouvalov. Le tableau 2 (voir l'annexe) montre que seuls le duc de Biron, le vicomte de Rochechouart et le duc de La Rochefoucauld, fils de la duchesse d'Enville, y apparaissent et encore cela n'est-il que pour une visite. En avril 1778, Denis Ivanovitch Fonvizine remarque cette tendance : « [...]

17. Voir le compte rendu du ballet dans Lesprit des journaux, août 1778, t. VIII, p. 284 .

18. [Nicolas-Toussaint Le Moyne Des Essarts], Causes célèbres, curieuses et intéressantes, de toutes les cours souveraines du royaume avec les jugements qui les ont décidées, Paris, Lacombe, 1777, t. XXXIV, p. 3-64.

19. Dans son ouvrage méticuleux basé sur les travaux du contrôle des étrangers, Antoine Lilti ne retient que les visites chez la comtesse Chouvalova (Antoine Lilti, Le monde des salons. Sociabilité et mondanité à Paris au XVIII siècle, Paris, Fayard, 2005, p. 129, tableau 1).

20. AMAE, Contrôle des étrangers, vol. 31, f. $83 v^{\circ}$; vol. 25 , f. $63 v^{\circ}$. 
de surcroît les dames françaises sont fières. Chouvalova va chez beaucoup d'entre elles, mais personne ne vient la voir ${ }^{21} »$. Ce qui relevait de la «fierté française » pour un observateur de l'époque peut recevoir une tout autre interprétation. Ne s'agit-il pas d'un exemple de cet échange culturel inégal entre la France et l'Europe discuté récemment à propos du théâtre par Rahul Markovits ${ }^{22}$ ? Le comte Andrei Chouvalov arrive à Paris pour se faire reconnaître, se divertir et surtout s'informer. Pour cela, il n'a pas forcément besoin de recevoir des Français chez lui, son intention étant de s'intégrer à la société parisienne.

Dans ce contexte, la présence de diplomates saxons, danois et autrichiens chez les Chouvalov attire notre attention. En 1778 et 1779, le prince Joseph Maria von Lobkowitz, ancien ambassadeur du Saint Empire en Russie, «fort lié23 » avec la famille russe, le baron Schoenfeld, ministre plénipotentiaire de Saxe en France, et le baron Blome, l'envoyé extraordinaire de Danemark en France, visitent tantôt la comtesse, tantôt le comte. Mais si les dîners de Ekaterina Petrovna sont suivis de toutes sortes d'amusements (des spectacles, des concerts de musique, de fréquentes promenades avec le prince Ivan Bariatinski dans le bois de Boulogne et aux environs de Paris), les soirées d'Andrei Petrovitch ont un tout autre but.

Il ne faut pas oublier que les années 1777-1781 sont marquées par deux guerres, celle de Succession de Bavière et celle de l'Indépendance des États américains, concernant de telle ou telle manière les intérêts de la Russie et de la France. En octobre 1778, Catherine II menace d'intervenir dans les deux conflits aux côtés des adversaires de la France et son ministre ne manque pas de l'annoncer haut et fort le 2 novembre 1778: «Il a été dit aujourd'hui entre ce ministre [de Russie] et le comte Schouwalow que la Russie fournissait 30 ooo hommes au Roi de Prusse; et 60 ooo hommes et beaucoup de vaisseaux à l'Angleterre $^{24}$ ». La situation se calme au printemps 1779 avec la médiation de la diplomatie russe que le prince Ivan Bariatinski discute pendant le

21. Denis Fonvizine, Lettres de France (1777-1778), traduites du russe et commentées par H. Grosse, J. Proust et P. Zaborov, préface de W. Berelowitch, Paris/Oxford, CNRS/Voltaire Foundation, 1995, p. 126.

22. Rahul Markovits, Civiliser l'Europe: politiques du théâtre français au XVIII siècle, Paris, Fayard, 2014.

23. AMAE, Contrôle des étrangers, vol. 32, f. $54 \mathrm{v}^{\circ}$.

24. Ibid., vol. 25 , f. $63 \mathrm{v}^{\circ}$. 
dîner chez le comte Chouvalov le 2 mars 1779. Laffaire se termine loin de Paris, par la paix de Teschen le 13 mai 1779, et devient l'une des voies de préparation pour la future alliance russo-autrichienne ${ }^{25}$. Cependant la présence du ministre de Saxe et de l'envoyé de Danemark dans la maison d'Andrei Chouvalov, aussi bien que l'apparition ultérieure (à la fin de 1779) du prince Lobkowitz, prouvent que le traité de Teschen et l'accord entre les deux empires se négociaient tant à Paris qu'à Vienne, Saint-Pétersbourg ou Berlin.

La maison du comte russe est à la fois un lieu de médiation diplomatique et de médisance politique. La police remarque, non sans un léger agacement, que la société d'Andrei Chouvalov se montre très anglophile à l'époque où la France mène la guerre contre son éternel ennemi. Lors des traditionnels dîners de mercredi, l'hôte de la maison et ses compatriotes «s'entretiennent avec beaucoup d'animosité des affaires de la France et de l'Angleterre, en prenant le parti de cette dernière puissance ${ }^{26} »$. Au total, on note six rapports mentionnant les nouvelles défavorables à la France, voire de fausses rumeurs en faveur de l'Angleterre. Sans oublier la menace de supporter l'Angleterre qu'annonce le prince Ivan Bariatinski le 2 novembre 1778, citons les autres cas flagrants. Le 30 décembre 1778, «M. le comte de Schouwalow raconte comme une nouvelle véritable, le soulèvement de deux colonies contre le Congrès ${ }^{27}$ ». Le 12 janvier 1780, «M. le ministre plénipotentiaire de Russie et ses compatriotes ont dîné chez M. le comte de Schouwalow, se sont beaucoup entretenus des affaires du temps, et ont marqué la plus grande satisfaction des avantages qu'ont eu les Anglais dans certaines circonstances ${ }^{28}$ ». Le 3 juin 1780, au dîner privé du comte Chouvalov, le prince Ivan Bariatinski, le baron Schoenfeld et le baron Blome discutent de la bataille entre l'escadre du comte de Guichen et la flotte britannique commandée par l'amiral Rodney ${ }^{29}$. Enfin, le 14 février 1781, Nikolai Khotinski, chargé d'affaires

25. Ibid., vol. 28 , f. $82 v^{\circ}$. Sur la médiation de la Russie pendant la guerre de Succession de Bavière, voir M. А. Петрова, Екатерина II и Иосиф II. Формирование российско-австрийского союза 1780-179o [M. A. Petrova, Catherine II et Joseph II. La formation de l'alliance russo-autrichienne 1780-179o], Moscou, Nauka, 2011, p. 117-121.

26. AMAE, Contrôle des étrangers, vol. 31, f. $83 v^{\circ}$.

27. Ibid., vol. 28 , f. $12 \mathrm{v}^{\circ}$.

28. Ibid., vol. 33 , f. $26 \mathrm{r}^{\circ}$.

29. Ibid., vol. 34 , f. $101 \mathrm{r}^{\circ}-\mathrm{v}^{\circ}$. 
russe, «a assuré chez M. le comte de Schouwalow qu'on avait reçu dimanche la nouvelle d'un combat entre un vaisseau anglais et un espagnol, que le premier avait perdu plus de cent hommes à l'abordage mais qu'il était cependant rendu maître de l'espagnol et que cette prise était extrêmement riche ${ }^{30}$ ».

Le cercle politico-mondain que crée chez soi le comte Chouvalov est ouvertement anglophile et implicitement anti-français. L'intrigue que mène ou aide à mener l'hôte de la maison implique soit les diplomates russes, formés à la cour de Saint-Pétersbourg dans les années 1760-1770 $\mathrm{O}^{31}$, soit les étrangers hostiles à la politique extérieure de la France, les Saxons, les Danois, les Autrichiens. Le comte Chouvalov lui-même n'est pas un aveugle instrument dans ce stratagème. Élève de Voltaire, factotum de Catherine II dans sa correspondance avec le patriarche de Ferney ${ }^{32}$, il doit partager les goûts politiques de sa souveraine et de son maître à penser et à écrire. Mais alors que le réseau anglophile fonctionne bien, le comte Chouvalov et ses habitués s'attirent le mécontentement du cabinet de Versailles et le mépris d'une bonne partie de l'aristocratie parisienne.

Dans la situation où certaines portes ne sont pas ouvertes, le comte Chouvalov et ses compatriotes cherchent des sources d'information variées. Il est intéressant de repérer dans cette compagnie le docteur Johann Gottfried ou Jean-Geoffroy Saiffert (Leipzig, 1747-Paris, 1810). Installé à Paris depuis 1777, ce médecin d'origine saxonne (introduit chez les Chouvalov par le baron Schoenfeld?) traite des gens de tous états, mais ses clients ordinaires sont le duc d'Orléans et la princesse

30. AMAE, Contrôle des étrangers, vol. 37, f. $64 v^{\circ}$.

31. Sur l'anglomanie en Russie sous Catherine II, voir Anthony Glenn Cross, "By the banks of the Thames »: Russians in Eighteenth Century Britain, Newtonville, Oriental Research Partners, 1980; Anthony Glenn Cross, «By the banks of the Neva»: Chapters from the Lives and Careers of the British in Eighteenth-century Russia, Cambridge, Cambridge University Press, 1997; А. А. Орлов, «Теперь вижу англичан вблиз... Британия и британцы в представлениях россиян о мире и о себе (вторая половина XVIII - первая половина XIX вв.). Очерки [A. A. Orlov, « Maintenant je vois les Anglais de près... " La Grande Bretagne et les Britanniques perçus par les Russes (seconde moitié du XVIII-première moitié du XIX siècle). Essais], Moscou, Giperboreia, Kučkovo pole, 2008, p. 13-74.

32. Sur «la correspondance à trois » (Voltaire - A.P. Chouvalov - Catherine II), voir Alexandre Stroev, «L'impératrice et le patriarche», Voltaire, Catherine II. Correspondance 1763-1778, éd. de A. Stroev, Paris, Non Lieu, 2006, p. 7-29 et suivantes. 
de Lamballe 33 . En février 1780, le ministre plénipotentiaire de Russie «va tous les jours» chez les Chouvalov «tant pour conférer avec le comte, que pour apprendre les nouvelles que M. Seiffer, médecin allemand recueille dans Paris et y apporte ${ }^{34}$ ». Politiques et mondaines à la fois, les anecdotes du docteur attirent non seulement les Russes, mais aussi les diplomates étrangers devenus les habitués de la maison: le baron Schoenfeld, le baron Blome et le prince Lobkowitz. Les informations semblent tellement importantes que, à en croire Saiffert sur parole, on lui propose de devenir le premier médecin de l'impératrice de Russie avec 50 ooo livres d'appointements et le rang de lieutenant-général ${ }^{35}$.

\section{Les enjeux mondains et politiques d'une poésie fugitive}

Vu l'intérêt que porte aux affaires politiques le comte Chouvalov, il est plus aisé d'expliquer sa volonté de plaire à la duchesse de La Vallière dont le salon est un vrai centre de la vie diplomatique dans le Paris prérévolutionnaire ${ }^{36}$. Dans le Mercure de France du 11 septembre 1779 apparaissent les Vers à Madame la duchesse de La Vallière, le jour de

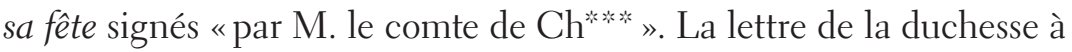
Ivan Ivanovitch Chouvalov datée du 23 janvier 1780 permet d'attribuer avec certitude cette poésie à Andrei Petrovitch Chouvalov: «M. le comte de Schowaloff a fait pour ma fête des vers très jolis, ils ont été imprimés dans le Mercure, si vous l'avez à Pétersbourg, vous les y aurez ${ }^{37} »$. Comme cette œuvre n'a jamais été liée au nom du poète francophone, nous nous permettons de reproduire ici ce texte:

33. Sur la clientèle de Saiffert et ses activités sociales et politiques voir Augustin Cabanès, La princesse de Lamballe intime (d'après les confidences de son médecin), Paris, Albin Michel, 1922.

34. AMAE, Contrôle des étrangers, vol. 33, f. $70^{\circ}$.

35. Augustin Cabanès, La princesse de Lamballe, op. cit., p. 32. Aucune autre source ne prouve cette déclaration du médecin.

36. Antoine Lilti dénombre 610 visites de diplomates étrangers chez la duchesse de La Vallière entre 1774 et 1789, un chiffre qui n'est dépassé que par les Necker qui reçoivent 640 visites (Antoine Lilti, Le monde des salons, op. cit., p. 130-131, tableau 1).

37. ОР РНБ [Département des manuscrits, Bibliothèque nationale de Russie], Fr. Q.IV.207, f. 163. La duchesse de La Vallière étant née le 11 décembre (1713), on peine à expliquer pourquoi le comte Chouvalov fait publier la poésie trois mois avant l'anniversaire. 
À vos amis vous êtes chère, Ils sentent le bonheur de vivre auprès de vous.

Les grâces et l'esprit ont un charme bien doux, Mais on ne plaît longtemps que par le caractère;

C'est un don fortuné qui les surpasse tous,

Et qui distingue la Vallière;

On adore son cœur, son goût et sa raison.

La touchante Beauté qui reçoit notre hommage,

De sa couronne, hélas! perd toujours un fleuron.

Le temps qui détruit son ouvrage,

En planant sur notre horizon,

Fait pâlir l'astre du bel âge.

Qui ne possède rien est alors isolé;

Il gémit en secret, et n'est pas consolé.

Ce deuil n'est point pour vous: Voltaire sur sa lyre

Chantait vos agréments et ne vous flattait pas;

Il fit votre Portrait, qu'on aime et qu'on admire;

Ce qu'il vantait, le temps ne saurait le détruire:

Quand on a l'art de plaire, on garde ses appas.

Ah! que souvent pour nous ce jour se renouvelle!

Que la santé se joigne à tous vos dons divers,

Que l'on chérisse en vous des grâces le modèle!

Puissiez-vous et passer l'âge de Fontenelle,

Et recevoir de meilleurs vers ${ }^{38}$ !

Cet exemple de poésie fugitive frappe par l'accent que met son auteur sur les qualités sociales de la destinatrice. L'incipit est explicite: le comte Chouvalov vise à établir le contact dès le début en soulignant "le bonheur de vivre auprès» de la duchesse que ressentent ses amis. D’après le poète russe, les grâces, le charme, le goût ne sont pas des dispositions naturelles chères en elles-mêmes. Elles font partie de ce précieux art de plaire et aident à résister contre la force dévastatrice du temps ( «Le temps qui détruit son ouvrage... / Qui ne possède rien est alors isolé»). Le thème de sociabilité que développe le comte Chouvalov oppose son œuvre au modèle choisi, le Portrait de Madame la Duchesse de La Vallière par Voltaire (vers 1744). Le philosophe brosse une image abstraite, un assemblage parfait de qualités personnelles:

38. Mercure de France, samedi 11 septembre 1779, p. 49-50. 
Etre femme sans jalousie,

Et belle sans coquetterie;

Et bien juger sans beaucoup savoir,

Et bien parler sans le vouloir;

N'être haute ni familière;

N'avoir point d'inégalité $[\ldots]^{39}$.

Le comte Chouvalov insiste sur le fait que les talents de la duchesse ont un impact social. Dans le Souhait, une autre pièce fugitive du poète francophone russe adressée à la princesse Natallia Kirilovna Zagriajskaia, née comtesse Razoumovskaia, l'opposition badine entre les grâces naturelles et l'art de plaire devient le sujet central :

Dieu charmant des plaisirs les plus doux de la terre,

Amour! Tu sais tout enflammer.

Donne-moi des leçons dans l'art touchant de plaire;

Ah! sans maître j’appris l'art facile d'aimer.

Daigne aussi, Reine de Cythère,

Guider Philis et la former.

Enseigne-lui l'art facile d'aimer;

Pour mon tourment elle a celui de plaire ${ }^{40}$.

L'imagerie et le ton léger de cette poésie (et des Vers à Madame la duchesse de La Vallière) rappellent plutôt la stylistique de Dorat que celle de Voltaire. Mais si dans les relations avec le patriarche de Ferney, le rôle de «filleul » littéraire est imposé au comte Chouvalov d'avance (rappelons que certains journalistes de l'époque croient fermement à ce que le poète francophone russe ne fait que prêter son nom à Voltaire, d'après leur avis auteur véritable de l'Epître à Ninon de l'Enclos), tout est plus compliqué avec Dorat. Le comte russe protège Dorat, comme il sied à un homme de son rang, l'imite, comme s'il était son disciple en poésie, et dialogue avec lui sur un pied d'égalité dans les journaux littéraires. Par cette variété d'attitudes, Andrei Chouvalov réalise ce qu'il ne pouvait pas se permettre avec Voltaire et espère ainsi compléter son image d'aristocrate éclairé et policé. Mais Dorat n’est pas un

39. Euvres de Voltaire, éd. de M. Beuchot, Paris, Firmin-Didot frères, 1833 , t. XIV, p. $387-388$.

40. Esprit des Journaux, octobre 1777, t. X, p. 243-244. La destinatrice de cette pièce a été identifiée par Dmitri Kobeko, dans «Élève de Voltaire », art. cit., p. 271. 
bon choix. Même son ami La Harpe lui trouve «l'esprit frivole et le goût faux ${ }^{41} »$.

La poésie «des plaisirs les plus doux de la terre » peut quand même plaire dans certains hôtels comme celui de la comtesse de Beauharnais ${ }^{42}$, mais non pas dans les cercles plus influents et plus sophistiqués où le comte Chouvalov aspire à s'installer. Compte tenu de l'opinion de la marquise Du Deffand, lisons l'avis de la duchesse de La Vallière. Le 23 août 1780, elle écrit à Ivan Ivanovitch Chouvalov pour gronder son neveu:

Mme la comtesse de Schowaloff jouit ici d'une grande considération, elle est aimée et estimée comme elle le mérite par tout le monde. Pour moi, elle a des bontés charmantes [...]. Je n'ai pas toutes les bonnes grâces de son mari, il a fait pourtant des vers pour moi qu'il a fait mettre dans le Mercure; mais les beaux esprits l'occupent trop pour perdre son temps dans les conversations frivoles ${ }^{43}$.

Le mot est lâché de nouveau: le beau monde parisien n’accepte pas «les beaux esprits». À la cour de Saint-Pétersbourg, on ne les apprécie pas non plus. Catherine II trouve les choix littéraires et sociaux du comte Chouvalov peu judicieux et exprime son mécontentement dans une missive destinée à Grimm. Elle écrit le 30 novembre 1778, quelques mois après l'échec des Barmécides de La Harpe: "Je suis fâchée que ce père du Désert se soit si opiniâtrement coiffé de M. de Laharpe, et je suis bien aise que malgré ses claquements, les Barmécides soient tombés, parce que cela rendra son excellence plus sage, s'il peut le devenir ${ }^{44} »$. Outre l'aspect moralisateur de ce propos ironique, notons aussi ses implications politiques. Dans les années 1770-1780, les relations entre Catherine II et son fils, et successeur présumé, le grand duc Paul, deviennent de plus en plus tendues. La tsarine regarde d'un très mauvais œil, en la soupçonnant d'un complot,

41. Jean-François de La Harpe, Lycée ou Cours de littérature ancienne et moderne, Paris, Deterville, 1818 , t. VIII, p. 261.

42. Sur les relations entre Dorat et la comtesse Fanny de Beauharnais, voir Gustave Desnoiresterres, Le chevalier Dorat et les poètes légers au XVIII siècle, Paris, Perrin, 1887 , p. 137-150 et suivantes; Eric Noël, Les Beauharnais: une fortune antillaise, 1756-1796, Genève, Droz, 2003, p. 207-212 et 215.

43. ОР РНБ [Département des manuscrits, Bibliothèque nationale de Russie], Fr. Q.IV.207, f. 163-164.

44. СИРИО [Sbornik imperatorskogo russkogo istoritcheskogo obstchestva], SaintPétersbourg, 1878, t. XXIII, p. 113. 
toute personne qui se rapproche du grand duc. Or La Harpe adresse au comte Chouvalov, entre 1774 et 1789 (avec un hiatus de cinq ans, 1777-1781, soit le temps du séjour parisien d’Andrei Petrovitch), une correspondance littéraire dont le vrai destinataire est Son Altesse Impériale ${ }^{45}$. Une fois de plus, les relations sociales et littéraires servent de cadre pour un jeu politique.

Les connaissances du comte Andrei Petrovitch Chouvalov dans les cercles littéraires, dont La Harpe et Dorat, peu prisés par le grand public, de même que ses relations scandaleuses avec la demoiselle Asselin, contrastent avec sa condition et provoquent une certaine contrariété tant chez la tsarine que chez les grandes dames parisiennes. N'oublions pas non plus les bruits politiques anti-français qui se répandent à travers sa maison. Soucieux de s'imposer à Paris en tant que «maître en l'art de plaire», le comte russe mérite le titre de «bel esprit» de la part des maîtresses des salons les plus influents. Comment expliquer ce paradoxe? Le cas du comte Andrei Petrovitch Chouvalov paraît bien représentatif des efforts de nombreux Parisiens russes au siècle des Lumières. Quelque francophiles qu'ils soient, ils tombent dans le piège, entre les deux cultures, et (modifions ici l'ordre des mots de Madame de Staël concernant le prince de Ligne ${ }^{46}$ ) deviennent imitateurs dans le genre français, au lieu d'en être modèles. Peut-être encore Ivan Ivanovitch Chouvalov égale-t-il l'habile «prince rose». Mais son neveu, qui choisit parfois de mauvais modèles littéraires à suivre, mêlé dans les intrigues anti-françaises de sa cour, reçoit un accueil bien mitigé.

45. Cette correspondance littéraire a été publiée par Christopher Todd en 1973 (Letters to the Shuvalovs, op. cit.). L'original se trouve à Saint-Pétersbourg: РО ИРЛИ [Département des manuscrits, Institut de littérature russe], f. 344 (Les Chouvalov), $\mathrm{n}^{\circ} 2$ (Lettres de La Harpe à Andrei Petrovitch Chouvalov).

46. Rappelons ce fameux jugement de Madame de Staël dans la Préface pour les lettres et pensées du prince de Ligne publiées en 1809: "Peut-être même le prince de Ligne est-il le seul étranger qui, dans le genre français, soit devenu modèle, au lieu d'être imitateur » (CEuvres complètes de Madame la baronne de Staël-Holstein, Paris, Firmin-Didot frères, 1836 , t. II, p. 260). 
AnNeXe 1

Tableau 1: Fréquentation des salons parisiens par les Chouvalov (d'après les rapports de police, 1777-1781)

\begin{tabular}{|c|c|c|c|c|c|c|c|c|c|c|c|c|c|}
\hline & 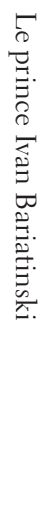 & 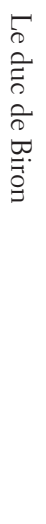 & 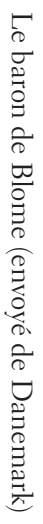 & 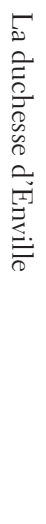 & 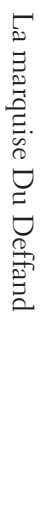 & 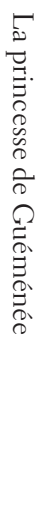 & 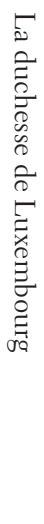 & 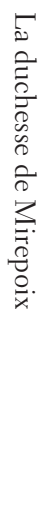 & 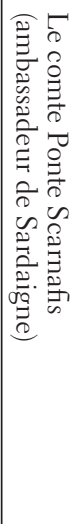 & 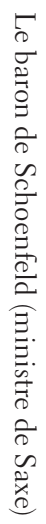 & 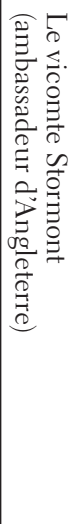 & $\begin{array}{l}5 \\
0 \\
0 \\
0 \\
0 \\
0 \\
0 \\
0 \\
0 \\
0 \\
0 \\
0 \\
0 \\
0 \\
0 \\
0 \\
0 \\
0 \\
0 \\
0\end{array}$ & 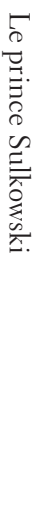 \\
\hline Les Chouvalov & 1 & 5 & 1 & & 2 & & & & 1 & 1 & 1 & 2 & 1 \\
\hline $\begin{array}{l}\text { Andrei } \\
\text { Chouvalov }\end{array}$ & & 3 & 1 & & 1 & & & & & & & & \\
\hline $\begin{array}{l}\text { Ekaterina } \\
\text { Chouvalova }\end{array}$ & 2 & 4 & & 1 & & 1 & 1 & 1 & & 3 & & & 2 \\
\hline
\end{tabular}

Annexe 2

Tableau 2: Fréquentation de la maison des Chouvalov à Paris par les Français et les étrangers (d'après les rapports de police, 1777-1781)

\begin{tabular}{|c|c|c|c|c|c|c|c|c|c|c|c|c|c|}
\hline & 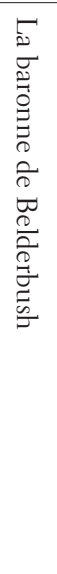 & 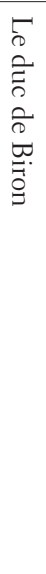 & $\begin{array}{l}5 \\
0 \\
0 \\
0 \\
0 \\
0 \\
0 \\
0 \\
0 \\
0 \\
0 \\
0 \\
0 \\
0 \\
0 \\
0 \\
0 \\
0 \\
0 \\
0 \\
0 \\
0 \\
0 \\
0 \\
0 \\
0\end{array}$ & 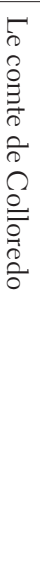 & 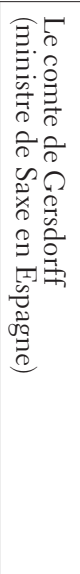 & 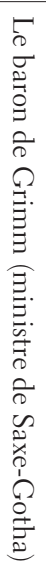 & 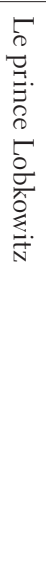 & 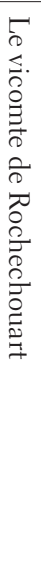 & 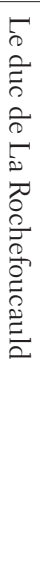 & 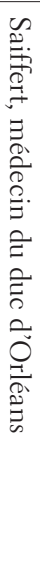 & 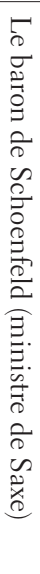 & $\begin{array}{l}5 \\
0 \\
0 \\
0 \\
0 \\
0 \\
0 \\
0 \\
0 \\
0 \\
0 \\
0 \\
0 \\
0 \\
0\end{array}$ & 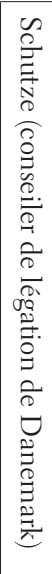 \\
\hline $\begin{array}{l}\text { Andrei } \\
\text { Chouvalov }\end{array}$ & & & 13 & & 3 & 3 & 2 & & 1 & 3 & 15 & 1 & 1 \\
\hline $\begin{array}{l}\text { Ekaterina } \\
\text { Chouvalova }\end{array}$ & 1 & 1 & 8 & 1 & & 9 & 5 & 1 & & & 14 & & 2 \\
\hline
\end{tabular}

